using Grading of Recommendations Assessment, Development and Evaluation.

Setting Recreational and elite sports settings or military settings.

Patients (or Participants) Athletes (all sports) and military personnel above 16 years of age.

Interventions (or Assessment of Risk Factors) Exposure: GJH or shoulder joint hypermobility.

Main Outcome Measurements Acute shoulder injury or activity-related shoulder pain.

Results In total, 2,496 participants $(31.9 \%$ females, mean age 19.9 years) from seven studies were included. Athletes with joint hypermobility were more likely to have shoulder injuries $\left(\mathrm{OR}=3.41,95 \% \mathrm{CI} 1.88,6.21, \mathrm{I}^{2}=71.5 \%\right)$ than athletes without joint hypermobility. Exposure definition had large impact on estimates (GJH, OR $=1.97$, 95\% CI 1.32, 2.94; shoulder joint hypermobility, OR $=6.79$, 95\% CI $3.91,11.80 ; \mathrm{p}=0.002)$, while remaining subgroup analyses showed no differences. The overall quality of evidence was low.

Conclusions We found 3-fold higher odds of shoulder injuries among athletes with joint hypermobility compared with nonexposed athletes. Due to low quality of evidence, future research may change the effect estimate. These findings highlight the need for prevention of shoulder injuries in athletes with joint hypermobility.

\section{THE IMMEDIATE EFFECTS OF SERVING ON SHOULDER ROTATIONAL RANGE OF MOTION IN TENNIS PLAYERS}

${ }^{1}$ Katy Williams, ${ }^{2}$ Clair Hebron. 'University of Bath, Bath, UK; ${ }^{2}$ University of Brighton, Brighton, UK

10.1136/bjsports-2021-IOC.87

Background A decrease in dominant shoulder internal and total rotational range of motion (ROM) has been found in tennis players immediately after competitive play. This can become a risk factor for shoulder injuries if certain thresholds are reached. The adaptation is theorised to result from follow through of the serve, requiring the shoulder to decelerate through eccentric action of external rotators. This theory has not been confirmed by empirical research comparing serving to groundstrokes.

Objective To investigate the immediate effects of serving on shoulder rotational ROM in tennis players by comparing to groundstrokes.

Design Same-subject, randomised, crossover design.

Setting International High Performance Tennis Centre.

Participants Eighteen male and 12 female professional and university tennis players.

Interventions Participants undertook both serving and groundstroke interventions (involving 120 tennis balls) in a randomised order.

Main Outcome Measurements Passive glenohumeral internal and external rotation ROM measurements, using a digital inclinometer, were undertaken at baseline and immediately following serving and groundstroke interventions on both dominant and non-dominant shoulders. Total rotation was calculated as the sum of internal and external rotation.

Results On the dominant and non-dominant shoulders there was no significant interaction effect between the factors of tennis intervention (serving and groundstrokes) and time (pre and post $(p=<0.05)$. Indicating that change in rotational ROM was not specific to tennis intervention. On the dominant shoulder there was a significant main effect of time $(p=$ 0.007), with internal, external and total rotational ROM decreasing irrespective of tennis intervention.

Conclusions Both tennis interventions resulted in immediate significant reductions in shoulder rotational ROM on the dominant shoulder but not the non-dominant shoulder of professional and university tennis players. These were within normal ranges that do not present risk factors for shoulder injuries. There was no significant difference between serving and groundstroke interventions. This might be an important consideration when planning tennis training.

\section{SWIMMING-RELATED COMPLICATIONS DURING TRIATHLON EVENT}

${ }^{1}$ Amilia Alifrangis, ${ }^{3}$ Martin Suzan, ${ }^{1,2}$ Courtney Kipps. 'University College London, London, UK; ${ }^{2}$ Institute of Sport and Exercise Health, London, UK; ${ }^{3}$ Swim Safety Ltd, London, UK

\subsection{6/bjsports-2021-IOC.88}

Background The majority of serious medical complications within triathlons occur during the swim leg of the race. Race organisers and medics have a duty of care to ensure that their teams are prepared to rapidly identify, remove and treat swimmers in distress.

Objective To describe the incidence and causes for water rescue team extractions from the swim part of triathlon events. Design Observational study.

Setting Two large community-based mass-participation triathlon races held in the UK during the summer over 4 years between 2013-2016.

Patients (or Participants) All participants requiring intervention from the water rescue team were included in the study. Relay participants were excluded.

Interventions (or Assessment of Risk Factors) Demographics including age, gender; and race factors including distance and wave size.

Main Outcome Measurements Participant demographics and reasons for intervention were recorded in water rescue team extraction logs. Outcomes of serious medical complications were recorded where available.

Results Race reports from 7 triathlons in the UK between 2013 - 2016 were analysed. 44,159 triathletes started the events, competing across swim leg distances of $400 \mathrm{~m}, 750$ $\mathrm{m}$ and $1500 \mathrm{~m} .232$ competitors required extraction from the water (5.2/1000 starters). Those aged 30-34 were most likely to require extraction. There was no significant difference in extraction rates between male and female participants. $14.7 \%$ of extractions were due to breathing difficulties. Other reasons for extraction were tiredness (31\%), cramp (12\%) and injury (8\%). Two male athletes suffered cardiac arrests in the water and required resuscitation. One later died in hospital.

Conclusions Serious medical complications in the swim leg of triathlons are rare but can be fatal. Water rescue and race medical teams must be adequately prepared to rapidly identify and intervene in such cases. Further research is needed to look at causes of breathlessness in injury in these events. 